\title{
A Hybrid Scheme in Cloud Computing for Secure Sharing of Data in the Cloud
}

\author{
Gopichand G, Koye Sai Vishnu Vamsi, Yarram Sai Subhash Reddy, Kolli Sai Poorna Chand, \\ Gaddipati Saiteja
}

\begin{abstract}
Cloud computing is rapidly getting to be a direct result of the provisioning of flexible, versatile, and on-ask for storing and figuring administrations for customers. In cloud-based capacity idea, information proprietor does not have full power over claim information since information is controlled by the outsider called cloud service providers (CSP). Information security is testing issue when information proprietor shares claim information to another entity known as information sharer on cloud. Numerous specialists have tended to this issue by using cryptography with various encryption plots, that give secure information sharing on cloud. Here, we propose framework for secure information sharing on cloud with intension to give information secrecy, get to control all of the offered information, expelling the weight of key administration and document encryption/unscrambling by clients, bolster progressively changes of client's participation and also working on providing information to the client whenever he/she needs it without the proprietor being constantly online to provide the same
\end{abstract}

Keywords: Cloud Computing, Data security, Cloud Service Providers (CSP), Secure Sharing, Cryptography, Access control

\section{INTRODUCTION}

The SeDaSC approach is proposed to give the flexibility and ease of use when it comes to securing the data sharing in the social networking and events related to the same, also providing confidentiality with respect to securing the data from the unauthorized insiders. This approach counters the data altogether and can give the data to the cloud users without the interference of the insiders. By virtue of SeDaSC, the report is encoded with key age calculation $(\mathrm{K})$. There is cryptographic server (CS) where key age calculation $(\mathrm{K})$ is done and is also deleted specifically after the completion of the process. This $\mathrm{K}$ can be created again the same by any client or even by the CS. For privacy, the information can't be released except if the aggressor accesses $\mathrm{K}$. K completely isn't put away anyplace, this K however doesn't even transfer from one place to another in the network. Subsequently, the generation of $\mathrm{K}$ in the network is kind of troublesome. Client shares can sometimes be grabbed by assailant also can be termed as Ki but other accuracy should be taken care properly. The figure or arbitrary age is to be produced using

Revised Version Manuscript Received on 10, September 2019.

Gopichand G, School of Computer Science and Engineering, Vellore Institute of Technology,Vellore, Tamil Nadu, India.

Koye Sai Vishnu Vamsi, School of Computer Science and Engineering, Vellore Institute of Technology, Vellore, Tamil Nadu, India.

Yarram Sai Subhash Reddy, School of Computer Science and Engineering, Vellore Institute of Technology,Vellore, Tamil Nadu, India.

Kolli Sai Poorna Chand, School of Computer Science and Engineering, Vellore Institute of Technology,Vellore, Tamil Nadu, India.

Gaddipati Saiteja, School of Computer Science and Engineering, Vellore Institute of Technology,Vellore, Tamil Nadu, India.

an aggregate of $2^{\wedge} 256-1$ conceivable offers. The likelihood of producing the right offer is $(1 /(2256-1))=8.636 \times$ $10^{\wedge}-78$, which is irrelevant. Also, on the off chance that the insider inside the cloud picks up entry of data, then the $\mathrm{K}$ should be the factor that should cause the hinderance and provide the mystery. Various keys concept isn't used in this approach because it can provide security while sharing the data. Symmetric key concept is used to do the encryption. Be that as it may, the approved clients are conceded access based on ownership of the key offer and the normal verification and approval marvel. The Access Control Link (ACL) records the approved clients with their qualifications while also comparing them with the key generated by the CS. Then the $\mathrm{K}$ is generated when the client is ready. A substantial client controls the rightful decoding of the data. Inside the social network this division and the dispersal of the key helps in countering the unauthorized customers and insiders. Each gathering document is different and provided by ACL. In this manner, a legitimate gathering client can't get to the gathering document that isn't imparted to him/her. An endeavor to get to an unapproved document is additionally obstructed by the way that the client won't have the key offer for that record. In addition, the ACL of the unapproved document won't contain any records for the malevolent client. Besides, the nonattendance of the whole key to the client and the ACL all things considered guarantees the forward and in reverse access control for the information. El-Gamal cryptosystem which also comes with bilinear coordinating is greatly responsible for sending the reliant data. Re-encryption of the data every time the path to the data is asked for is initiated mostly by clients/customers rather than insiders/proprietor. The El-Gamal cryptosystem is real when it comes to computation. Moreover, overhead is added when re-encryption is done for each path. The SeDaSC framework is different as it uses symmetric encryption, also the path and social network is also handled by the customers as explained previously. Compared to El-Gamal the total overhead for the approach is petty less

\section{RELATED WORK}

Md Mozammil et al. [5]: The cell phone is utilized for transferring, downloading and sharing of information yet it has restricted limit of calculation. thus, when versatile client need to share possess cell phone information to another on cloud by secure way can take after the proposed arrangement 
by this scientist where information proprietor encodes the information utilizing blowfish calculation which is quick and required little measure of memory which is reasonable for cell phones and sends it to cloud stockpiling. The information proprietor sends email of scrambled document to the sharer at that point secretly give mystery key to the information sharer. Sharer decodes the document got in mail utilizing mystery key and gets the first information.

Uma et al. [6]: In Cloud computing keep up information privacy, confirmation and uprightness is principle issue when information sharing happens with someone else on cloud. in this way, according to proposed arrangement by scientist's message process of plain content is marked by proprietor with RSA calculation and plaintext message is encoded by people in general key of beneficiary. Beneficiary will decode the figure content to plaintext with his private key, and from that register the message process code, which is contrast and the seared message process code by proprietor if both are indistinguishable then mark is substantial, and information say information share safely. This system tackles the issue of information secrecy, validation and trustworthiness.

Mazhar et al. [2]: For share information in aggregate on cloud get to control of client, forward and in reverse mystery issue is comes which is illuminated by specialists. They have proposed SeDaSC technique by presenting CS (cryptographic server), encryption/unscrambling activities. At the point when client need to transfer/download the mutual record on cloud joins claim mystery key given by CS and CS will takes the proper activities on the plaintext/figure content document. The proposed SeDaSC gives privacy of information, safely share information, get to control of client and control the forward and in reverse access.

Ching-Hung et al. [10]: Using open key cryptography PKI share information in gather is awkward and if utilize private key cryptography key dispersion is principle issue and furthermore take care of the issue of forward and in reverse mystery. According to proposed display by specialists for secure information sharing on cloud Only 1 open key which is regular in assemble utilizing that encode information which need to share by any gathering part and another part in gathering can get that information utilizing own private key which is allotted by amass pioneer. Here, Group pioneer takes all obligations of key age and refreshing it when any part leaves or join/rejoin the gathering pioneer refresh open and private key.

Criteria in view of underneath rundown of written works audit table is developed are, information Confidentiality for secure private information and just verified clients can demonstrate it, information uprightness checking instrument give on information sharer side ,information sharing model reasonable is appropriate for information share in gathering or distributed, get to rights which are relegated by proprietor to sharer for information on cloud is just perused or all (read, compose, erase and so on.) and proprietor ought to be constantly on the web or not when the client needs to get to the information.

Sharing of sensitive data in the cloud can also be seen carried out in the El-Gamal cryptosystem as mentioned by Khan [2], here the approach is panned out in such a way that all the task that are key generation, encryption, re-encryption, etc is given to an outsiders who controls all of this and this also brings into picture the bilinear complexations and mixing.

Chen and Tzeng [9] proposed an approach related to symmetric key technique considering the inference technique for anchoring information sending among a gathering. For the keys they have used a double true. This approach is costly, and the re-keying adds to more overhead. Additionally, the approach isn't custom fitted for open cloud frameworks claiming specific tasks require incorporated interventions. A comparable RSA (Rivest Shamir Adleman) based technique was additionally introduced. Be that as it may, the plan was powerless against intrigue assaults.

\section{SYSTEM ARCHITECTURE}

The SeDaSC technique works with three parameters Users; A cryptographic server (CS); and the cloud; users. The CS is a basic parameter used in securing the key and it oversees key association with respect to the network. The same key or the symmetric key is passed through by CS and the data within is divided with the help of key. Thus, in the network for each customer, the CS divides the key into two segments with the true objective that a singular part alone can't recoup the key. Dynamically, the principal key $\mathrm{K}$ is terminated through secure overwriting. The ACL has one part of the key while the other part is transmitted with the key. The ACL is used in the network to provide data to the requesting customers/clients. ACL acts as the intermediate for data transfer in the network. The data is stored in the cloud in an encrypted format. A downloaded demand is sent to CS from the network to access the data, this can be sent by the owner or a requesting client. The information of the records present in the cloud can be downloaded with the help of CS, where the bit of the key is used which is found with the client. The key can be recovered or rediscovered by the bit present with the client and that specific client can be identified looking into the CS. The information is sent back to the customer/client once the data is unscrambled. The client is added to the ACL after joining of both the bits of the key is done. When the information id delivered it is erased from ACL and the network. The leaving part can't decipher the data in solitude as he/she simply has a touch of the key. Consequently, no progressive unscrambling and re-encryption are required if there ought to emerge an event of changes in the social occasion enlistment. Additionally, SeDaSC used with the flexible distributed computing perspective despite conventional distributed computing because of the way that procedure concentrated assignments are performed by the CS.

\section{PROPOSED SYSTEM \& RESULTS}

Here we propose a philosophy named Secure Data Sharing in Clouds (SeDaSC) those courses of action with the recently referenced security essentials of shared assembling data inside the cloud. The SeDaSC procedure works with three components as takes after: 
1) Clients

2) a cryptographic server (CS) and

3) The information owner of the cloud presents the information, the outline of the purchasers, moreover, the parameters needed for creating a path management list (ACL) to the network. The CS is a basic parameter used in securing the key and it oversees key association with respect to the network. The same key or the symmetric key is passed through by $\mathrm{CS}$ and the data within is divided with the help of key. Thus, in the network for each customer, the CS divides the key into two segments with the true objective that a singular part alone can't recoup the key. Dynamically, the principal key $\mathrm{K}$ is terminated through secure overwriting [10]. The ACL has one part of the key while the other part is transmitted with the key. The ACL is used in the network to provide data to the requesting customers/clients. Encoded information is in like manner exchanged to the cloud for limit considering a legitimate concern for the customer. Whenever the data is needed to a requesting customer, it triggers the CS. A section of the key $\mathrm{K}$ is present with the customer/client, so to download the data report which is present in the cloud the requesting customer uses this section with the help of CS.

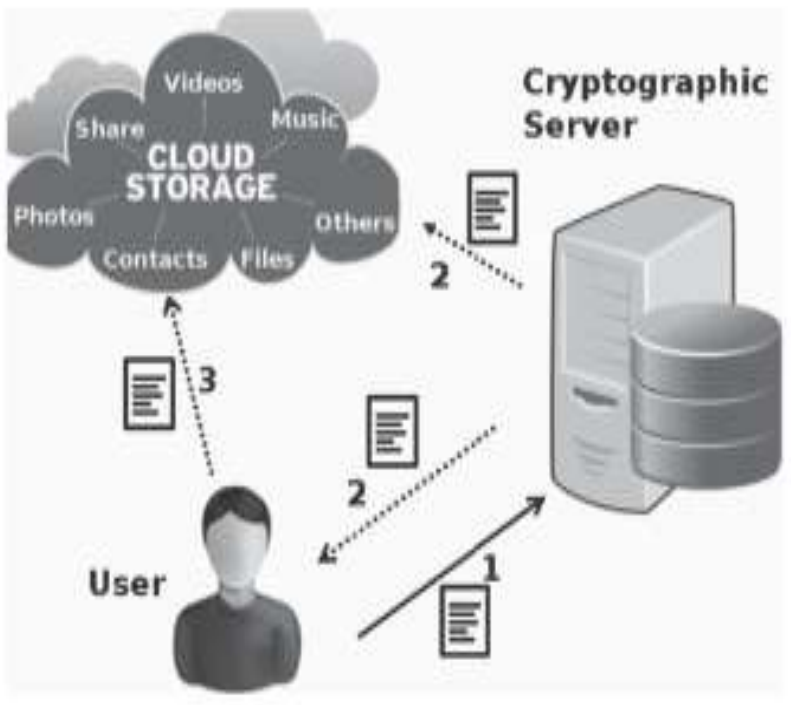

Fig.1 Architecture

\section{A. Algorithm:}

Stage 1: PSEUDONYM GENERATION: THE PSEUDONYM GENERATION ALGORITHM IS RUN BY EACH USER. Information: ID Output: Pseudonym P

Stage 2: Convergent encryption:

Key-Gen-CE (M) - Here the term K refers to the key used for age computation of the data which also maps the data or information that is present in the copy $\mathrm{M}$ to the key $\mathrm{K}$ being generated.

Enc-CE (K, M) - Here term $\mathrm{C}$ refers to the same key encryption estimation which takes information from the key $\mathrm{K}$ and the $\mathrm{M}$ which is the data copy, and from $\mathrm{M}$ the figure content $\mathrm{C}$ is yielded.

$\operatorname{Dec}-\mathrm{CE}(\mathrm{K}, \mathrm{C})$ - Here term $\mathrm{M}$ refers to the count which is calculated by both key $\mathrm{K}$ and the figure content $\mathrm{C}$. With these parameters the data copy $\mathrm{M}$ can be yielded.

\section{CONCLUSION}

Among them, the homomorphism key understanding grants affirmed customers acquire control enter thus as to achieve the inspiration driving record sharing. The difference between SeDaSC system and the others is that the process of encryption and the functionalities related to unraveling the data are altogether performed at the CS level. The system which is proposed can also be used to versatile conveyed figuring because of the way in which that method concentrated undertakings are performed at the CS. We delineated the assents given by the administrations, their semantics, and the passageway giving techniques that are used to apply these approvals to customers. Likewise, a course of action of traditions for sharing data securely in a couple of open amassing mists was displayed. These traditions were by widening an ideal plan of properties required for sharing data between customers of a cloud benefit.

\section{REFERENCES}

1 Abbas and S. U. Khan, "A review on the State-of-the-art privacy preserving approaches in e-health clouds," IEEE J. Biomed. Health Informat., vol. 18, no. 1, pp. 14311441, Jul. 2014.

2 N. Khan, M. M. Kiah, S. A. Madani, M. Ali, and S. Shamshir-band, "Incremental proxy re-encryption scheme for mobile cloud computing environment," J. Supercomput., vol. 68, no. 2, pp. 624-651, May 2014.

3 L. Wei, H. Zhu, Z. Cao, Y. Chen, and A. V. Vasilakos, "Security and privacy for storage and computation in cloud computing," Inf. Sci., vol. 258, pp. 371-386, Feb. 2014.

4 K. Alhamazani et al., "An overview of the commercial cloud monitoring tools: Research dimensions, design issues, stateof-the-art," Computing, DOI 10.1007/s00607-014-0398-5, 2014, to be published.

5 D. Chen et al., "Fast and scalable multi-way analysis of massive neural data," IEEE Trans. Comput., DOI: 10.1109/TC.2013.2295806, 2014, to be published.

6 S. Seo, M. Nabeel, X. Ding, and E. Bertino, "An Efficient Certificateless Encryption for Secure Data Sharing in Public Clouds," IEEE Trans. Knowl. Data Eng., vol. 26, no. 9, pp. 2107-2119, Sep. 2013.

7 N. Khan, M. L. M. Kiah, S. U. Khan, and S. A. Madani, "Towards secure mobile cloud computing: A survey,' Future Gen. Comput. Syst., vol. 29, no. 5, pp. 1278-1299, Jul. 2013.

8 L. Xu, X. Wu, and X. Zhang, "CL-PRE: A certificateless proxy reencryption scheme for secure data sharing with public cloud," in Proc. 7th ACM Symp. Inf. , Comput. Commun. Security, 2012, pp. 87-88

9 Y. Chen and W. Tzeng, "Efficient and provably-secure group key management scheme using key derivation," in Proc. IEEE 11th Int. Conf. TrustCom, 2012, pp. 295-302.

10 S. U. R. Malik, S. K. Srinivasan, S. U. Khan, and L. Wang, "A methodology for OSPF routing protocol verification," in Proc. 12th Int. Conf. ScalCom, Changzhou, China, Dec. 2012, pp. 1-5.

11 Cloud security Alliance, "Security guidelines for critical areas of focus in cloud computing v3.0," 2011. 
12 L. Moura and N. Bjrner, "Satisfiability modulo theories: An appetizer," in Proc. Formal Methods, Found. Appl., vol. 5902, Lecture Notes in Computer Science, 2009, pp. 23-36.

13 P. Gutmann, "Secure deletion of data from magnetic and solid-state memory," in Proc. 6th USENIX Security Symp. Focusing Appl. Cryptography, 1996, p. 8.

14 T. Murata, "Petri Nets: Properties, analysis and applications," Proc. IEEE, vol. 77, no. 4, pp. 541-580, Apr. 1989.

15 Y. Chen, J. D. Tygar, andW. Tzeng, "Secure group key management using unidirectional proxy re-encryption schemes," in Proc. IEEE INFOCOM, pp. 1952-1960.

16 Santhi H, Gopichand G, Gayathri P, Automated Smart Parking System using IoT, Journal of Advanced Research in Dynamical \& Control Systems, Vol. 10, 09-Special Issue, 2018

17 P Gayathri, Mayank Agarwal, H Santhi, Gopichand G,Bone Breakage Identification Using Image Processing Techniques, Journal of Advanced Research in Dynamical \& Control Systems, Vol. 10, 09-Special Issue, 2018 